\section{The risks of defensive (emergency) medicine. The laboratory perspective}

\author{
Martina Montagnana, Giuseppe Lippi \\ Section of Clinical Biochemistry, \\ University of Verona, Verona, Italy
}

\section{Abstract}

Diagnostic testing is a crucial aspect of the clinical decision making, especially in emergency settings where timely and accurate diagnoses are essential for appropriate patient management. Reliable statistics attest that the vast majority of clinical decisions for diagnosis, treatment and follow-up of both acute and chronic diseases are influenced by results of laboratory analyses. As specifically concerns the emergency department, many unnecessary laboratory tests are also ordered in this healthcare setting, with unfavorable consequences on laboratory and healthcare organization. As far as the laboratory environment is concerned, defensive (emergency medicine) may be associated with incremental costs, derangement of laboratory organization, enhanced complexity of data management process, diagnostic delay attributable to performance of unnecessary testing, and litigation. Educative or even regulatory interventions are hence urgently needed to addresses problems of the current liability system, in order to decrease the detrimental effects of defensive (emergency) medicine in the laboratory.

\section{Introduction}

Diagnostic testing is a crucial aspect of the clinical decision-making. Reliable statistics attest that up to $70 \%$ of the clinical decisions for diagnosis, treatment and follow-up of both acute and chronic diseases are influenced by results of laboratory analyses, especially in emergency settings where timely and accurate diagnoses are essential for appropriate patient management. This categorical evidence poses a huge pressure on modern clinical laboratories, since these are forced to face a constant increase of type and volume of tests. Two independent surveys based in the USA and Italy recently showed that urgent testing represents a major part of the laboratory workflow, accounting for as many as $50 \%$ of all tests performed in clinical laboratories. ${ }^{1,2}$ In another study carried out in 36 different Spanish hospitals, Salinas and colleagues ${ }^{3}$ also showed that the rate of stat tests request was highly heterogeneous, ranging from 44 to 412 per
1000 emergency department (ED) patient admissions.

Due to the need of reporting urgent diagnostic information to clinicians and emergency physicians, stat analyses need to follow specific pathways within the laboratory, namely characterized by a shorter turnaround time compared to conventional testing. This obviously carries substantial organizational and economical consequences. Due to their peculiar characteristics, stat analyses have been for long performed using separate instrumentation, thus requiring dedicated personnel and incremental costs for purchasing specific analyzers and reagents. However, due to the growing diffusion of automation in clinical laboratories, the workflow of urgent testing has remarkably changed in the past decade, leading the way to two paradoxically opposite organizational solutions. ${ }^{4}$ In some laboratories urgent testing has now been consolidated within the so-called corelabs, i.e., aggregation of many analytical platforms performing different types of analyses and physically connected by belts or other sample conveyors, in which both stat and routine specimens can be loaded simultaneously, giving priority to those test requiring urgent notification. In other facilities, urgent testing has been instead decentralized in peripheral wards, namely EDs and intensive care units (Figure 1). Both solutions have advantages and limitations. Despite seldom plagued by a longer turnaround time, the former approach is indeed more economically attractive. Conversely, the use of point of care (POC) instrumentation in clinical wards allows a rapid release of test results, but obviously necessitates more expansive POC analyzers and reagents to be placed at the site of testing, along with an appropriate education of both nurses and physicians to run and ensure quality management of this peculiar type of analyses. Regardless of the way stat testing is performed, urgent testing remains a critical issue for modern healthcare systems.

\section{Opinion Report}

Defensive medicine has become a major driver of laboratory activity and healthcare economics, wherein many and seldom expensive tests are requested against evidence-based practice or violating well established criteria of appropriateness, thus leading to the so-called over-testing syndrome..$^{5-7}$ In a survey performed on a random sample of physicians practicing in six high risk specialties, ${ }^{8}$ Studdert and colleagues reported that $59 \%$ of physicians ordered unnecessary diagnostic tests or procedures due to defensive medicine. Another recent survey of the Italian Agency for Regional Healthcare Systems (Agenzia
Correspondence: Giuseppe Lippi, Section of Clinical Biochemistry, University of Verona, via delle Menegone, 37134 Verona, Italy.

E-mail: giuseppe.lippi@univr.it; ulippi@tin.it

Key words: Defensive medicine; Emergency medicine; Emergency department; Laboratory testing; Diagnosis.

Received for publication: 12 October 2015. Accepted for publication: 31 December 2015.

This work is licensed under a Creative Commons Attribution 4.0 License (by-nc 4.0).

(C) Copyright M. Montagnana and G. Lippi, 2016 Licensee PAGEPress, Italy

Emergency Care Journal 2016; 12:5581

doi:10.4081/ecj.2016.5581

Nazionale per $i$ Servizi Sanitari Regionali; AGENAS) based on over 1500 hospital physicians revealed that $58 \%$ of them admitted to practice defensive medicine and, even more importantly, as many as $93 \%$ of hospital physicians were not considering of suspending or reducing this practice. ${ }^{9}$ It is also noteworthy that laboratory tests were ranged first among the various diagnostic investigations requested for defensive reasons.

As specifically regards the ED, many unnecessary laboratory tests are also ordered in this healthcare setting, with unfavorable consequences on laboratory and healthcare organization. ${ }^{10}$ Beside defensive medicine, several additional reasons have been identified underneath the behavior of inappropriate laboratory tests request, including the broad use of multitest profiles, the diffusion of organ- or diseasespecific test panels, the request of tests not really necessary or redundant, obsolete tests analyses and excessive reporting delays. ${ }^{11,12}$

A paradigmatic example of misuse of laboratory test ordering can be identified in the diagnostic approach to patient with chest pain. Despite the current guidelines for management of patients with unstable angina and non ST-segment elevation myocardial infarction (STEMI) recommend to use only cardiospecific troponins for the identification of myocardial injury, ${ }^{13,14}$ and it also has been clearly demonstrated that the use of high-sensitivity cardiac troponins in a patient presenting with chest pain is accurate enough to rapidly rule-out or rule-in AMI, ${ }^{15}$ many emergency physicians still request a large number of creatine kinase isoenzyme MB (CK-MB) tests in association with troponin. According to this evidence, different institutions have eliminated CK-MB from emergency panel tests in the past years. Interestingly, Singh et al. reported that the elimination of CK-MB from the diagnostic panel for patients with suspected acute coro- 
nary syndrome (ACS) was associated with a considerable drop of requests for this biomarker, from approximately 12,000 to 150 per year, with no effect on the diagnostic accuracy. ${ }^{16} \mathrm{Le}$ and colleagues recently reported the results of a real world experience at Brigham and Women's Hospital, Boston, MA, USA. ${ }^{17}$ All cases with normal values of troponin $\mathrm{T}(\mathrm{TnT})$ and increased concentration of CK-MB were reviewed, but no discrepancy between values led to impairment of the ACS diagnosis. Conversely, the elimination of CK-MB from routine ED test menu was associated with cost savings of approximately $\$ 47,000$.

The introduction of thyroid analyses (i.e., thyroid-stimulating hormone; TSH) in the ED test panels is another controversial issue. The clinical utility may be somehow justified in rare clinical conditions such as myxedema coma and thyroid storm, which are potentially lethal. ${ }^{18-20}$ Atrial fibrillation (AF), the most frequently for of arrhythmia observed in ED, can also be triggered by hyperthyroidism. ${ }^{21}$ However, the real need to perform urgent TSH testing is highly questionable, and each circumstance should be accurately weighted against the need of preventing unnecessary costs. Buccelletti and colleagues ${ }^{21}$ carried out a cross-sectional observational study, in which TSH was measured in all patients admitted to the ED for new-onset AF over a 30 months period. The results of the study showed that a clinical model incorporating a history of previous thyroid disease, stroke/transient ischemic attack and hypertension was effective to identify the presence of hyperthyroidism with a remarkably high (i.e., 0.93) sensitivity, thus making TSH testing virtually meaningless. Bellew and colleagues studied 1964 consecutive patients receiving a final diagnosis of $\mathrm{AF}$ in the ED. ${ }^{22}$ Decreased (i.e., $<0.3 \mathrm{U} / \mathrm{mL}$ ) and enhanced ( $>5 \mathrm{U} / \mathrm{mL}$ ) TSH values could only be observed in 2 and $11 \%$ of patients, respectively, thus confirming that TSH measurement may not be cost-effective in the ED. ${ }^{22}$ More recently, Giacomini and colleagues assessed the clinical usefulness of TSH test requests performed in patients admitted to $\mathrm{ED},{ }^{23}$ and also found that elevated and decreased TSH values were only present in 3 and $1.2 \%$ of the patients, respectively. In only one patient displaying an increased TSH value the measurement of this biomarker led to a substantial change of the clinical decision making due to the presence of Hashimoto thyroiditis.

Despite the diagnostic value of lipase is higher than that of amylase, the concomitant request of both biomarkers is still commonplace in the ED for the diagnosis of acute pancreatitis. ${ }^{24}$ Nevertheless, it has been demonstrated that the urgent, simultaneous assessment of both amylase and lipase does not significantly modify the accuracy of the diagnosis. ${ }^{25-27}$ Indeed, the foremost limitation of amylase is represented by the low specificity for diagnosing pancreatitis, since increased values are observed in a kaleidoscope of diseases affecting the biliary tract, liver, intestines, genitourinary tract, lungs, breast, prostate, central nervous system and salivary glands. Moreover, the diagnostic sensitivity of amylase was also found to be lower than that of lipase,$^{28}$ wherein normal values of serum amylase can be present in as many as $30 \%$ of patients with acute alcoholic pancreatitis. ${ }^{29}$ It is also noteworthy that lipase values persist elevated for a long time after pancreatitis, whereas amylase tends to normalize very rapidly, thus lowering its diagnostic sensitivity. As for CK-MK, it has hence been concluded that amylase testing does not provide add value over lipase for initial assessment of patients with suspected pancreatitis in the ED. ${ }^{30}$

\section{Conclusions}

Squeezed between lower economical resources, increased volumes of testing and enhanced healthcare expectations, clinical laboratories are now facing a dedicate balance between efficiency and efficacy. ${ }^{31}$ Recent statistics attests that defensive medicine consumes 23 to $67 \%$ of laboratory resources, so that this form of inappropriateness ends up to magnify an already delicate equilibrium. ${ }^{32}$ Although the (unfavorable) clinical implications arising from defensive (emergency) medicine will be further discussed in another article published in this Journal, its negative impact on the organization of clinical laboratories is undeniable (Table 1), especially if one considers the high volume of urgent testing

Table 1. Negative impact of defensive (emergency) medicine on the organization clinical laboratories.

\begin{tabular}{ll} 
No. & Negative impact \\
1 & Incremental costs \\
2 & Derangement of laboratory organization \\
\hline 3 & Enhanced complexity of data management process \\
4 & Diagnostic delay attributable to performance of unnecessary testing \\
\hline 5 & Controversies and risk of false positive results \\
\hline
\end{tabular}

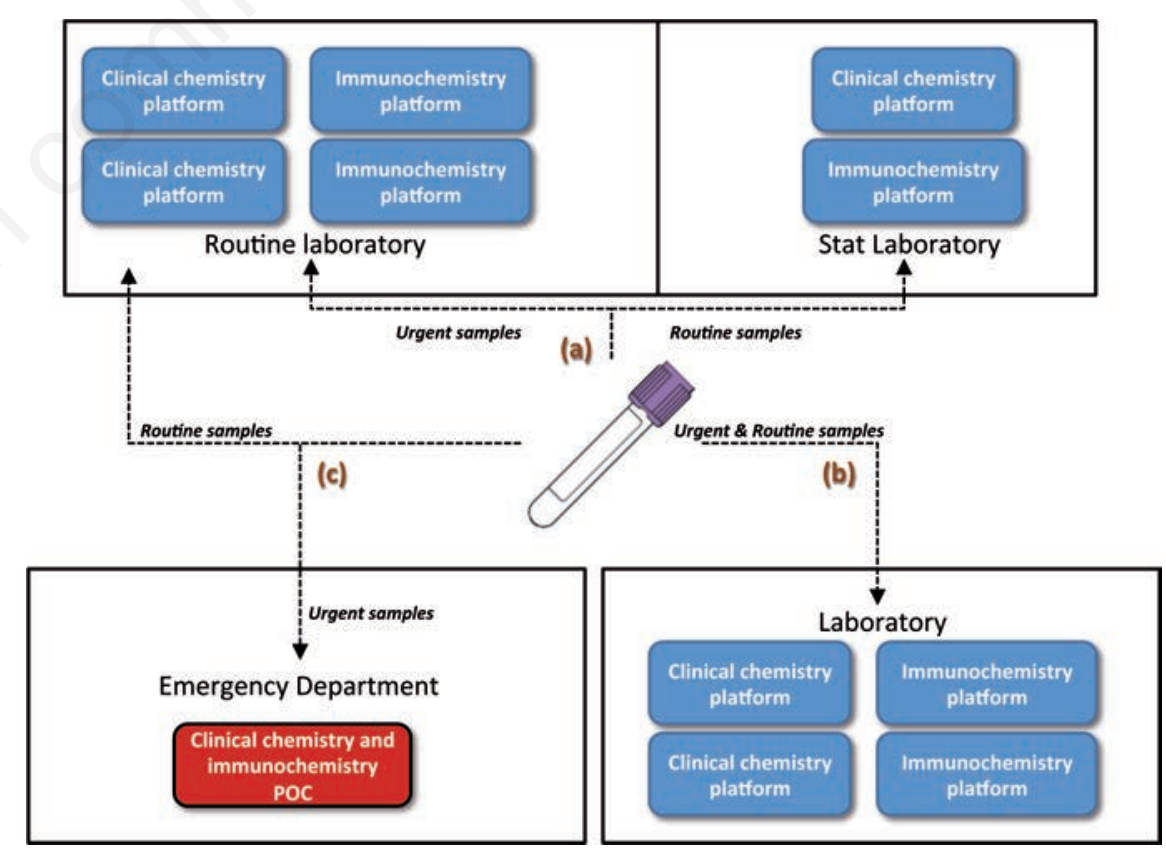

Figure 1. Organizational solutions for stat testing in clinical laboratories. a) Physical separation between routine and urgent testing; b) consolidation of routine and urgent testing; c) urgent testing performed with point of care (POC) instrumentation and routine testing carried out in the laboratory. 
currently carried out in clinical laboratories. As such, educative or even regulatory interventions are urgently needed to address problems in the current liability system in order to decrease the detrimental effects of defensive (emergency) medicine in the laboratory. ${ }^{33}$

\section{References}

1. Volmar KE, Wilkinson DS, Wagar EA, Lehman CM. Utilization of stat test priority in the clinical laboratory: a College of American Pathologists q-probes study of 52 institutions. Arch Pathol Lab Med 2013;137:220-7.

2. Lippi G, Mattiuzzi C, Plebani M. Stat testing utilization in clinical laboratories. National survey of Italian Society of Clinical Biochemistry and Molecular Biology (SIBioC). Clin Chem Lab Med 2014;52:79-84.

3. Salinas M, López-Garrigós M, Uris J, Pilot Group of the Appropriate Utilization of Laboratory Tests (REDCONLAB) Working Group. Differences in laboratory requesting patterns in emergency department in Spain. Ann Clin Biochem 2013;50:353-9.

4. Lippi G, Simundic AM, Plebani M. Phlebotomy, stat testing and laboratory organization: an intriguing relationship. Clin Chem Lab Med 2012;50:2065-8.

5. Zhi M, Ding EL, Theisen-Toupal J, et al. The landscape of inappropriate laboratory testing: a 15-year meta-analysis. PLoS One 2013;8:e78962.

6. Hoffman JR, Kanzaria HK. Intolerance of error and culture of blame drive medical excess. Brit Med J 2014;349:5702.

7. Rogg JG, Rubin JT, Hansen P, Liu SW. The frequency and cost of redundant laboratory testing for transferred ED patients. Am J Emerg Med 2013;31:1121-3.

8. Studdert DM, Mello MM, Sage WM, et al. Defensive medicine among high-risk specialist physicians in a volatile malpractice environment. J Am Med Assoc 2005;293: 2609-17.

9. Agenzia Nazionale per i Servizi Sanitari Regionali. Medicina difensiva: quanto ci costa. Available from: http://www.agenas.it/medicina-difensivaquanto-ci-costa
10. Trisorio Liuzzi MP, Attolini E, Quaranta R, et al. Laboratory request appropriateness in emergency: impact on hospital organization. Clin Chem Lab Med 2006;44:760-4.

11. Hindmarsh JT, Lyon AW. Strategies to promote rational clinical chemistry test utilization. Clin Biochem 1996;29:291-9.

12. Plebani M. Exploring the iceberg of errors in laboratory medicine. Clin Chim Acta 2009;404:16-23.

13. Thygesen K, Alpert JS, Jaffe AS, et al. Third universal definition of myocardial infarction. J Am Coll Cardiol 2012;60:1581-98.

14. Braunwald E, Antman EM, Beasley JW, et al. ACC/AHA guidelines for the management of patients with unstable angina and non ST-segment elevation myocardial infarction: executive summary and recommendations. A report of the American College of Cardiology/American Heart Association Task Force on Practice Guidelines (committee on the management of patients with unstable angina). Circulation 2000;102:1193-209.

15. Lippi G. Biomarkers: novel troponin immunoassay for early ACS rule-out. Nat Rev Cardiol 2016;13:9-10.

16. Singh G, Baweja PS. Creatine kinase-MB: the journey to obsolescence. Am J Clin Pathol 2014;141:415-9.

17. Le RD, Kosowsky JM, Landman AB, et al. Clinical and financial impact of removing creatine kinase-MB from the routine testing menu in the emergency setting. Am J Emerg Med 2015;33:72-5.

18. Bahn RS, Burch HB, Cooper DS, et al. Hyperthyroidism and other causes of thyrotoxicosis: management guidelines of the American Thyroid Association and American Association of Clinical Endocrinologists. Endocr Pract 2011;17:456-520.

19. Pimentel L, Hansen KN. Thyroid disease in the emergency department: a clinical and laboratory review. J Emerg Med 2005;28:201-9.

20. Mitchell JM. Thyroid disease in the emergency department. Thyroid function tests and hypothyroidism and myxedema coma. Emerg Med Clin N Am 1989;7:885-902.

21. Buccelletti F, Carroccia A, Marsiliani D, et al. Utility of routine thyroid-stimulating hormone determination in new-onset atrial fibrillation in the ED. Am J Emerg Med
2011;29:1158-62.

22. Bellew SD, Moman R, Lohse CM, et al. Validation of a decision rule for selective TSH screening in atrial fibrillation. West $\mathrm{J}$ Emerg Med 2015;16:195-202.

23. Giacomini A, Chiesa M, Carraro P. Urgent thyroid-stimulating hormone testing in emergency medicine: a useful tool? J Emerg Med 2015;49:481-7.

24. Vissers RJ, Abu-Laban RB, McHugh DF. Amylase and lipase in the emergency department evaluation of acute pancreatitis. J Emerg Med 1999;17:1027-37.

25. Keim, V, Teich, N, Fiedler, F, et al. A comparison of lipase and amylase in the diagnosis of acute pancreatitis in patients with abdominal pain. Pancreas 1998;16:45-9.

26. Yadav D, Agarwal N, Pitchumoni CS. A critical evaluation of laboratory tests in acute pancreatitis. Am J Gastroenterol 2002;97: 1309-18.

27. Werner H, Steinberg WM, Pauley C. Strategic use of individual and combined enzyme indicators for acute pancteatitis analyzed by receiver operator characteristics. Clin Chem 1989;35:967-71.

28. Orebaugh SL. Normal amylase levels in the presentation of acute pancreatitis. Am J Emerg Med 1994;12:21-4.

29. Spechler SJ, Dalton JW, Robbins AH, et al. Prevalence of normal serum amylase levels in patients with acute alcoholic pancreatitis. Dig Dis Sci 1983;28:865-9.

30. Volz KA, McGillicuddy DC, Horowitz GL, et al. Eliminating amylase testing from the evaluation of pancreatitis in the emergency department. West J Emerg Med 2010;11:344-7.

31. Lippi G, Mattiuzzi C. The biomarker paradigm: between diagnostic efficiency and clinical efficacy. Pol Arch Med Wewn 2015; 125:282-8.

32. Lippi G, Favaloro EJ, Franchini M. Dangers in the practice of defensive medicine in hemostasis testing for investigation of bleeding or thrombosis: part I. Routine coagulation testing. Semin Thromb Hemost 2014;40:812-24.

33. Plebani M. Defensive medicine and diagnostic testing. Diagnosis 2014;1:151-4. 